\title{
Enabling personalised medical support for chronic disease management through a hybrid robot-cloud approach
}

\author{
Laura Fiorini $^{1}$ - Raffaele Esposito ${ }^{1}$ - Manuele Bonaccorsi ${ }^{1}$. Claudio Petrazzuolo ${ }^{2}$. \\ Filippo Saponara $^{2}$ - Roberta Giannantonio ${ }^{2}$. Gianluca De Petris ${ }^{2}$ - Paolo Dario ${ }^{1}$. \\ Filippo Cavallo ${ }^{1}$
}

Received: 10 September 2015 / Accepted: 15 June 2016 / Published online: 8 July 2016

(C) The Author(s) 2016. This article is published with open access at Springerlink.com

\begin{abstract}
Information and Communication Technology and personal robots could play a fundamental role in efficiently managing chronic diseases and avoiding improper medications. They could support senior citizens with reminders, thus promoting their independent living and quality of life, especially in the presence of several chronic diseases (multimorbidity). In this context, this article proposes a service model for personalised medical support that is able to provide adequate healthcare service by means of a hybrid robot-cloud approach. This service was quantitatively and qualitatively tested to assess the technical feasibility and user acceptability level of the service. The service was tested with 23 older people (65-86 years) in the DomoCasa Lab (Italy). This study demonstrated the feasibility of the proposed hybrid cloud solution and the usability and acceptability were positively evaluated thus confirming the ability to utilise these innovative technologies for active and healthy ageing.
\end{abstract}

Keywords Cloud Robotics - Assistive Robotics - Ageing Well · Reminder Service · Quality of Service · Acceptability Assessment

\section{Introduction}

In Europe, persons over 65 years of age comprise $22.7 \%$ of the total population, which is expected to double in the next few years, thus increasing the demand for sustainable healthcare systems (EU 2013). This will increase the demand

\section{Filippo Cavallo}

f.cavallo@sssup.it

1 The Biorobotics Institute, Scuola Superiore Sant'Anna, Viale Rinaldo Piaggio 34, 56025 Pontedera, PI, Italy

2 Telecom Italia, WHITE Lab (Wellbeing and Health Innovative Technologies Lab), Via Cardinale Maffi 27, 56126 Pisa, Italy for nurse practitioners (+94\% in 2025) (Auerbach 2012) and physician's assistants (+72\% in 2025) (Hooker et al. 2011) with numerous implications for quality of care and for the organisation of future cost-effective care delivery systems. On the other hand, a decrease in the worker population (EU 2013) and a reduction of funds for social-medical services due to the current economic crisis will not fulfil the demand for care. Therefore, it is more likely that society has to sustain high costs to guarantee a high-level of assistance and Quality of Life (QoL) to senior citizens by providing medical cures, socio-medical and home care services.

Ageing causes a physiological decrease of motor, sensory and cognitive abilities in people. Older persons have a higher risk than the average population to suffer from chronic diseases, meaning that their independence may be reduced. A further complicating factor is the increasing prevalence of multiple concurrent chronic diseases (multimorbidity) (Marengoni et al. 2011). Moreover, EU senior citizens prefer to remain in their familiar home environments and maintain adequate QoL for as long as possible rather than to move to institutionalised care arrangements (Socio Political Observatory 2011). Two of the most common predictors of an older adult's transition to an assisted living facility are difficulty in efficiently managing medications and personal health management (Lieto and Schmidt 2005). Most older persons, when affected by one or several chronic diseases, may frequently require taking different drugs, performing specific therapies, periodically monitoring health parameters and the adoption of a specific and healthy lifestyle. These care procedures are often complex and can induce mental confusion in senior patients. Additionally, consequences of improper medications can be serious, contributing to $33-69 \%$ of hospital admissions (Osterberg and Blaschke 2005). Moreover, to better manage polypharmacy, older persons need to be supported and helped with their treatments (AGE 2010). For 
this reason, research efforts have mainly focused on medication adherence procedures such as convenient care, education programmes, counselling, reminders, self-monitoring and family therapy (Haynes et al. 2008).

In this context, Information and Communication Technology (ICT) and robotic technologies can make a large positive difference in the lives of older adults, including people with problems in everyday functioning and cognitive impairments (Wahl et al. 2012), and potentially lead to the development of a new care process (Moschetti et al. 2014). The application of these technologies represents a profitable solution to meet the physical and psychological needs of the older population. Recently, robotic technologies have become more common in older persons' daily life from both acceptability and usability points of view (Taipale et al. 2015; Moschetti et al. 2014; Coradeschi et al. 2011). Recent research (Solis and Carlaw 2013) has highlighted how robots for home healthcare applications represent the second major potential market opportunity for personal robotics. The large market size of domestic robots is mainly due to the demand for assistance robots in household tasks and those who will act as companions. In this context, Europe is expected to have the second-largest market for service robots. Robots have the potential to assist and support older adults in medication management needs, thus helping them lead a longer independent life in their own house. In particular, researchers' efforts have been focused on the design of novel services to accomplish different daily activities based on the integration and cooperation of companion robots and other agents such as smart environments (Tiwari et al. 2010). Many research groups and companies have developed robotic solutions, which could support both older people and the rest of the population during daily activities such as participation in performing specific tasks (Iwata and Sugano 2009; Mukai et al. 2008), acting as companion robots (Stiehl et al. 2005; Zhang et al. 2008), or providing complex assistive services (Meyer et al. 2009; Badii et al. 2009).

Recently, the fast growing cloud-computing paradigm (Sinčák et al. 2015), which allows users to use scalable and flexible computing resources, has led to the development of novel cloud robotics (Kamei et al. 2012; Goldberg 2014) and healthcare paradigms of services (Smith et al. 2013), which implement innovative strategies to serve the complex health needs of the ageing population.

These cloud-hybrid services overcome the increasing demand of nurse practitioners and physicians, who do not have sufficient resources and time to address all issues in standard appointments and help formal and informal caregivers in the delivery care process, supporting and enhancing the continuity of care (Fig. 1). Additionally, they potentially enable profitable relationships between patients, family members, caregivers, pharmacists and physicians, thus reducing the problems of management and medication treatments. Indeed the current medication procedures involve several actors, such as physician, pharmacist, caregiver and the user and their family, who essentially are not able to communicate with each other, thus performing different tasks independently. During the visit, the doctor interacts only with the user, and the pharmacist interacts only with the user or the caregiver. This means that the doctor-pharmacist interaction is almost absent; the relationships between caregiver and pharmacist and between doctor and patient are reduced in time and quality. Sometimes, doctors do not have sufficient time to address all issues in standard appointments (Healthdesk 2014).

In this sense, the gap between quality and duration of the relationships is at the origin of the problems of management and medication treatment. The user needs to feel in contact with all other actors involved: the caregiver, the local community and others stakeholders are needed to increase the duration and the intensity of their relationships.

Therefore, this article proposes a service model for personal health management that is able to provide adequate service both from users' and multi-stakeholders' points of view by means of a hybrid robot-cloud approach. The older person is at the centre of this service model surrounded by families, service providers, developers of ICT solutions, the local community and healthcare providers who are all collaborating to guarantee an adequate level of service. This proposed model provides a personalised medical support system in which one caregiver is able to manage more older people at the same time, providing the best care service without job role reduction. The caregiver and family manage and control the pharmacy adherence of several users by means of synergic action of cloud resources and a personal robot. Furthermore, a web portal can connect the pharmacist and the doctor. In addition, robotics and cloud resources open the possibility to design new services and tools to help service providers optimise their job.

\subsection{Related works}

The current commercial drug automatic dispensers (e-Pill; Philips; PivoTell) or talking pillboxes are limited in providing efficient reminder services. These solutions alone are not sufficient to enable active interaction, to invoke affect and social/physical support and to be connected in realtime with other stakeholders (Tiwari et al. 2011). During the last few years, several studies have shown how companion robots could be useful in providing reminders about medicine. Particularly, Prakash et al. 2013) underlined how users prefer robotic assistance to human assistance for medicine reminders. Their proposed system includes a personal robot (PR2), which acts as a physical reminder. However, this system does not include the collection of user feedback regarding the correct drug consumption. (Datta et al. 
Fig. 1 Ambient, user, robot and sensor with cloud and Software as a Service (SaaS)

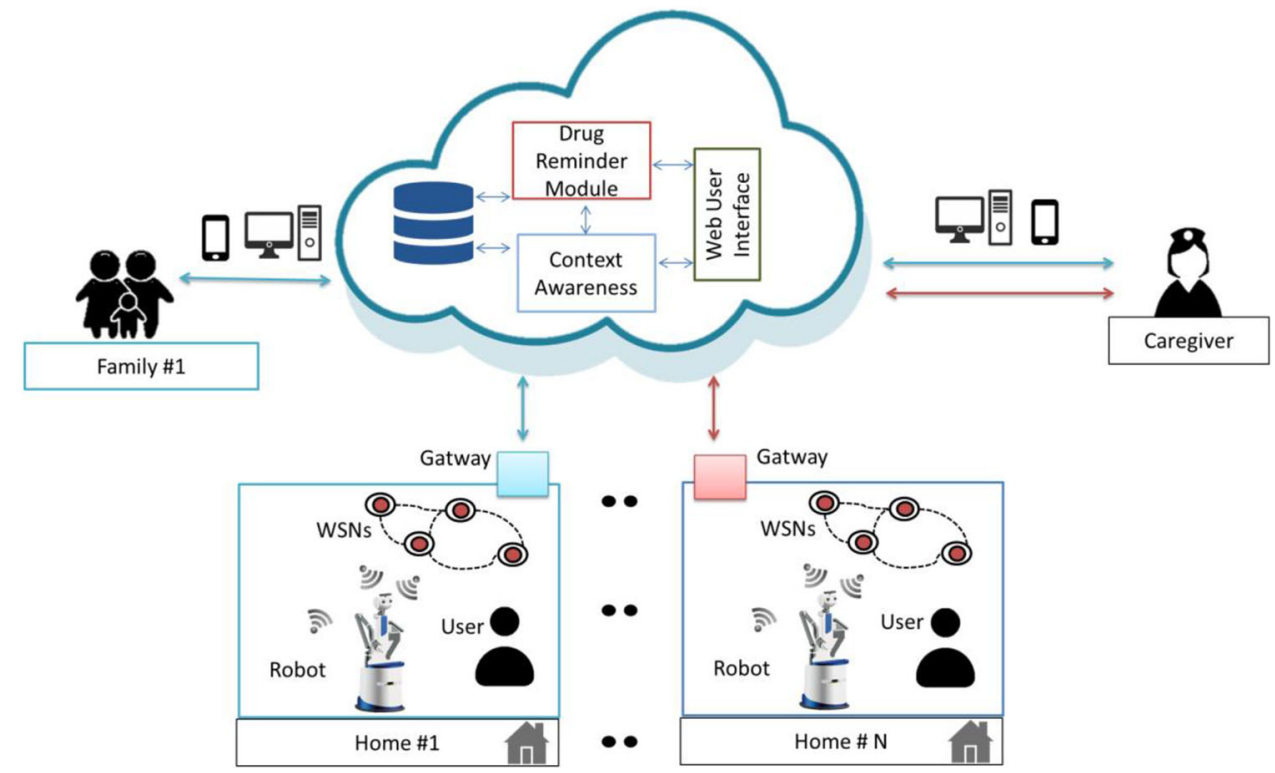

2011) designed a closed-loop medication management system using a healthcare robot with a web-based application (RoboGen) available $24 \mathrm{~h}$ per day, 7 days per week using any browser. This system included a personal robot with a touch screen, which was used to give users visual and auditory instructions, was able to collect user feedback and make them available through a web portal for other stakeholders. However, since the computational resources were stored in the robot, the service modularity and the robot capabilities were limited. In addition, recent work (Kaerlein 2015) and experimentation results (Tiwari et al. 2011) have provided guidelines for the design of robotic services concerning the simplification of the robots and the user interface to avoid misunderstanding and ambiguities.

In this context, this paper sought to improve this field by implementing a personal health management service, which combines the use of cloud computing services (both commercial and custom), a domestic robot, an android robot app and a web portal. The cloud software includes the indoor user localisation algorithm, and the voice recognition and speech synthesis tools. Furthermore, it is worth mentioning that this system was designed with special attention to state of the art guidelines concerning robot design, velocity and user interface (Prakash et al. 2013; Tiwari et al. 2011). The Domestic Robot, called DoRo, was developed under the Robot-Era project (Robot-Era) with a user-centre design approach to increase user acceptance. Its velocity was controlled in order to guarantee safety to the user. In addition, the robot app was designed to avoid errors and to help the user in recognising the correct drug consumption, thus increasing the system usability.

Eventually, this work also sought to qualitatively and quantitatively test the technological solution with real users in realistic scenarios to assess feasibility, acceptability and usability levels. In this study, the authors proposed a 'Reminder Service' to help older people with chronic disease management by means of a service based on a cloud-robot hybrid approach.

In this proposed service, the robot autonomously reached the user with the pre-set reminder event acting as a physical reminder and sending feedback on the correct consumption. The caregiver could access the web portal anywhere in order to set and check the reminder event after authentication procedures. The service was active $24 \mathrm{~h}$ per day.

The proposed system distributes the workload for the reminder service among a group of agents and cloud computing resources. The user localisation algorithm and the reminder module are stored in the cloud infrastructure, so different agents could be aware of the user's position. A database stores all the reminding information and the status of the house.

The first objective of this study was to design a modular service, which has the potential to be adaptable in different environments between different users. Secondly, a quantitative and qualitative service analysis was performed in terms of service efficiency, service reliability, service acceptability and service usability.

\section{Methods}

\subsection{Experimental setting and recruitment}

The evaluation of the proposed service's performance was based on a specific experimental protocol, which envisaged the involvement of senior citizens, the use of a realis- 
Fig. 2 DomoCasa experimental settings: the 4 main positions ( $A$ dining, $B$ kitchen, $C$ bedroom, $D$ bathroom, $O$ Robot starting point) and the environmental sensors. The two coloured circles are temperature, humidity and light sensors, the squares are PIR sensors, the octagons are presence sensors, the triangles are gas sensors and the circles are the switch sensors on the main door. In this figure, the user localisation network is not shown; for further details see Cavallo et al. 2014b)

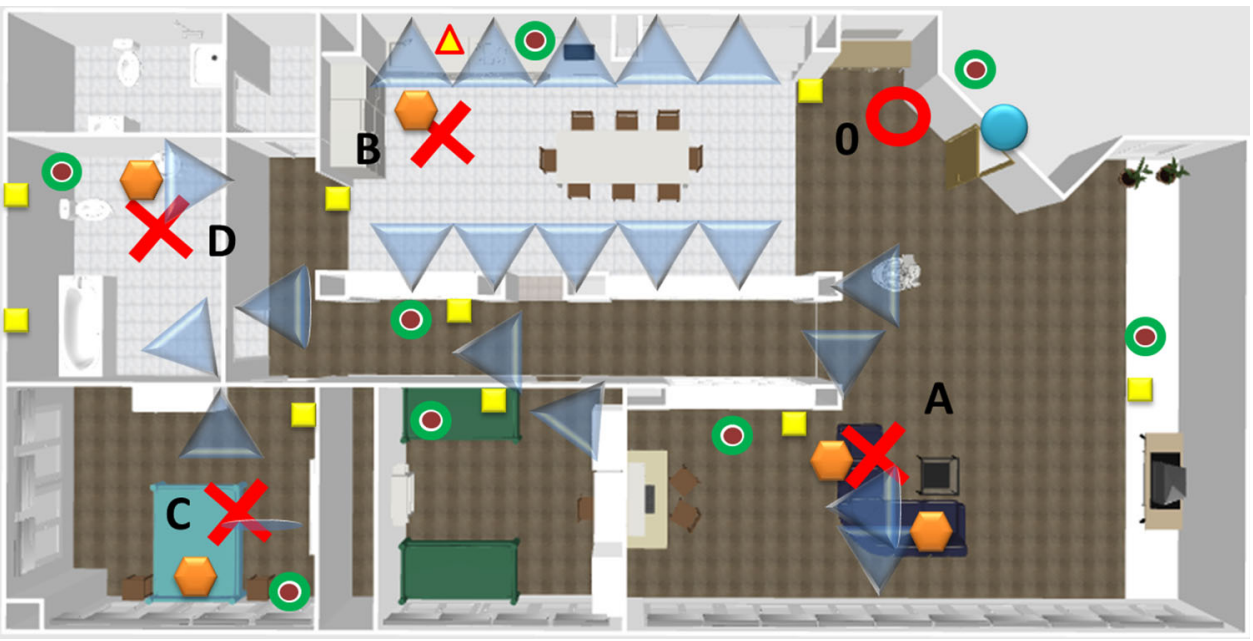

tic living lab and the measurement of appropriate metrics including the reliability and robustness of the system architecture and the acceptability and usability of the entire service.

The proposed service was realistically tested in the DomoCasa Lab (Peccioli, Italy), which reproduces a fully furnished apartment of 200 sqm with a living room, a kitchen, a bathroom and two bedrooms. DomoCasa was equipped with a mobile robotic platform, 20 localisation sensors, 9 proximity sensors, 5 sensorised carpets and pillows, 8 temperature, humidity and light sensors and 1 gas sensor. Furthermore, a gateway was used to gather sensor data to send to the cloud. Particularly, in the experimental trials, four rooms were identified in the DomoCasa (A-Living, B-Kitchen, C-Bedroom, D-Bathroom) (Fig. 2). In each room, a fixed position was used, respectively, on the sofa, in front of the sink, on the bed and in front of the washbowl. The mobile robotic platform was programmed to always start from the same position (Fig. 2, Position 0) with a pre-set maximum velocity of $0.6 \mathrm{~m} / \mathrm{s}$. These choices were made in order to have a standardised protocol to evaluate qualitative and quantitative parameters.

In order to assess technical feasibility and the acceptance of the service from real end-users, two different experimental sessions were conducted. For technical feasibility, the entire system was first tested without the presence of users for 5 consecutive trials for each of the 4 main selected positions A, B, C, D (in total 20 trials). In these trials the robot always starts from position 0 (charge station); a reminder event was set through the web portal and at the proper time the robot reaches the position (A, B, C, D). Finally a feedback was given using a proper app installed on the robot tablet to test the complete service (See Sect. 2.3).

For the acceptability assessment, the second experimental session was conducted with 23 older adults ( 15 females and 8 males), whose ages ranged from 65 to $86(73.45 \pm 6.27)$.
Before starting the study, all participants signed an informed consent form. All participants lived independently in their own apartments, $11(47.83 \%)$ with his/her spouse and 12 $(52.17 \%)$ lived alone. Regarding educational level, 7 participants $(30.43 \%)$ had a low educational level, attending the junior or junior high school, while $16(68.57 \%)$ had completed high school or university. All participants were independent in activities related to daily living. Users were asked to first compile the reminder event through the webportal ("Cassiel"), and after that were asked to move to one of the selected points. The complete scenario implemented during the experimentation is explained in Table 1. A researcher was present at all times and ready to intervene when needed. At the end of the trial, the user was asked to fill out a questionnaire to assess the acceptability and usability of the proposed service.

\subsection{Metrics and statistical analysis}

Metrics for assessing technical feasibility Quantitative data on the service's time was used to assess an acceptable Quality of Service (QoS) level. The performance of the proposed system was estimated through the following time measurements:

1. The Round Trip Time (RTT) - the time [ms] required for a signal pulse or packet to travel from a specific source to a specific destination and back again (Rouse et al. 2014). In this case, it was the time the system took to request the position of the user through the cloud.

2. The Robot Processing Time (RPT) - the time [ms] that the robot took to plan a reaction after receiving the input from the cloud. In this case, it was the time required to plan the path to reach the user after receiving the user's coordinates. 
Table 1 Description of the drug reminder application scenario in which the robot acts as a physical mediator

\begin{tabular}{ll}
\hline Actor & Actions \\
\hline End-user & $\begin{array}{c}\text { End-user remotely accesses the web portal, compiles and activates the drug reminder, then moves inside } \\
\text { the DomoCasa (in position A, B, C or D) } \\
\text { The sensor network continuously transmits sensor data to the gateway, which immediately forwards them } \\
\text { to the cloud database }\end{array}$ \\
Smart environments & $\begin{array}{l}\text { In the cloud, the module measures and stores the user's position in the database } \\
\text { At the time of the reminder event, the web application asks for the user localisation service in the cloud. } \\
\text { As soon as it receives the information, it sends a command to the robot to activate the physical reminder } \\
\text { Web application } \\
\text { The robot receives the command and plans the trajectory to reach the user (its on-board capabilities). } \\
\text { When in front of the user, the robot talks with him/her with natural language and, then a pop-up on the } \\
\text { graphical interface appears with the image of the medicine } \\
\text { The user takes the medicine and sends, through the app, feedback to the caregiver. This feedback is } \\
\text { available on the web-portal }\end{array}$ \\
\hline
\end{tabular}

3. Average Travel Time (ATT) - the time that the robot took to reach the user and provide the demanded service. This parameter was strictly correlated with the environment, particularly with the travel distance required to reach the user and the maximum velocity of the robot to move in the home (in this case $0.6 \mathrm{~m} / \mathrm{s}$ ).

4. The Total Service Time (TST) - service responsiveness is one of the key features that influences the acceptability and usability of the service's levels to improve the service efficiency (Cavallo et al. 2014a). It is computed as the sum of RTT, RPT and ATT.

5. Mean Velocity - represents the average velocity of DoRo. It was computed as the ratio between the distance and the TST.

Metrics for assessing the acceptance of the service Appropriate questionnaires were provided to users after testing the service to measure a quantitative metric of usability and acceptability. Usability can be defined as how effectively, efficiently and satisfactorily a user can interact with a technology and it is an important aspect for acceptability.

Acceptability is defined as 'the demonstrable willingness within a user group to employ technology for the tasks it is designed to support' (Dillon 2001). One of the first models developed to study the acceptance of the technology by an individual was the Technology Acceptance Model (TAM) (Davis 1989), based on Theory of Reasoned Action (Ajzen and Fishbein 1980) and Theory of Planned Behaviour (Ajzen 1991). The TAM is based on the perceived usefulness and perceived ease of use constructs, which determine attitudes to adopt new technologies. This model has been applied to the processes of adoption and use of many technologies, even if the social and normative variables are not taken into account. In order to provide a unified theoretical basis from which to facilitate research on technology adoption, the Unified Theory of Acceptance and Use of Technology (UTAUT) (Venkatesh et al. 2003) was developed.
The UTAUT is the most frequently used model for technology acceptance evaluation and was developed to investigate technology acceptance in workplaces (Heerink et al. 2006, 2009) found that the UTAUT model was not suitable for service robotics in domestic environments and suggested the need to adapt it for assistive social robots, developing consequently a new questionnaire, in which each construct was represented by multiple statements (Brooke 1996).

However each evaluation tool should be customised so the participants do not have trouble reading the questionnaire and making sure it appropriately suits the experiment aims. For these reasons in this paper, starting from the model proposed by Heerink et al., some questions were added. The complete list is shown in Table 3. Furthermore the Perceived ease of use construction was not suitable for this study so it was omitted. Apart from Heerink's model, two new constructions, Usability and Learnability, were added. The usability construction includes the System Usability Scale (SUS) (Brooke 1996). The SUS, which consists of ten questions, is a standardised tool for measuring usability, while the learnability is the capability of the proposed system to enable the user to learn how to use it. So a specific questionnaire for the hybrid robotcloud service was designed and applied in order to assess the acceptability of the proposed system.

Each user involved in the experimentation was asked to complete two different questionnaires: firstly, a sociodemographic questionnaire was provided before the experiment to collect information on socio-demographic data and technology experience; secondly, users answered the ad-hoc questionnaire, including SUS, on a five-point Likert scale (Strongly disagree (SD), Disagree (D), Neither agree nor disagree (NAND), Agree (A), Strongly agree (SA)).

The analyses included the calculation of Cronbach's Alpha in order to verify the reliability of the questionnaire (a reliable construct should have an Alpha value at least 0.7) (Santos 1999). Then a unique score, ranging from 0 to 100 was calculated and the scores were classified into three main 
Fig. 3 Overview of the system architecture with the different layers

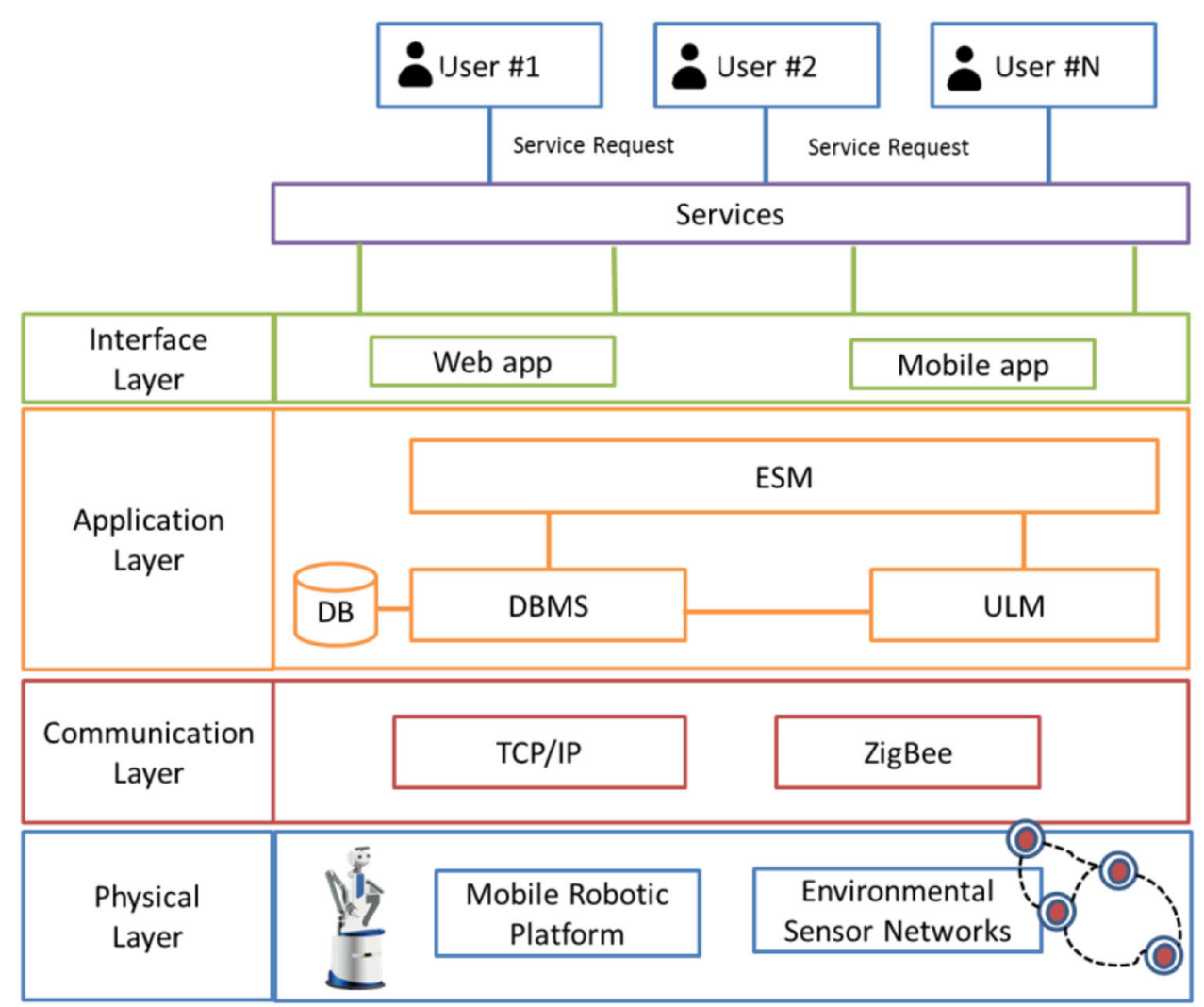

categories: not good (score $<65)$, good $(65 \leq$ score $<85)$ and excellent (score $\geq 85$ ) (McLellan et al. 2012). At a later stage a correlation analysis was conducted in order to verify the relations between the constructions. Furthermore the MannWhitney $\mathrm{U}$ and the Kruskal-Wallis tests were applied in order to compare different conditions and users. Finally in order to evaluate how each construct was related to other constructs, a correlation analysis was conducted. A p-value less than 0.05 was deemed statistically significant.

\subsection{System architecture}

According to the modular architecture (Fig. 3), the overall system integrates a group of hardware agents, i.e. the physical layer, which operates in the environment, and a cloud platform, i.e. the application and interface layers, which runs software tools for coordinating and providing the reminder service to users. The physical layer includes two sensor networks installed for both monitoring the environment and locating the user, and a mobile robotic platform (DoRo) equipped with a tablet, acting in the environment as a physical interactive mediator with the user.

The cloud platform was implemented through a database, used for collecting and storing data related to the environment and user, a localisation algorithm to know the position of the user within the environment, an event scheduler module devoted to planning and supervising the reminder service, and some human robot interface based on a web portal and app for direct interaction. This integration of physical agents with the cloud platform empowers the robot to outsource part of the software in the cloud (Hu et al. 2012 et al.; Kehoe et al. 2015; ROS) and allows for the design of flexible and modular services (Kamei et al. 2012).

Physical Layer The smart environment included two different sensor networks: one for user localisation using the Received Signal Strength (RSS) (Bonaccorsi et al. 2015), and one for environmental monitoring and passive localisation of the people (Cavallo et al. 2014a). The localisation network included a set of ZigBee Anchors placed in fixed positions in the environment, a ZigBee Coordinator to maintain and manage the entire network, a Data Logger (DL) and a Mobile Node (MN) worn by the user. Each anchor computed the RSS on the received messages from the MN. Similarly, the environmental network included a set of sensor nodes containing a selection of passive infrared (PIR) pressure sensors, switches on doors, windows and drawers, and temperature, humidity and light sensors. The two sensor networks were set on different channels to avoid interference and to ensure proper bandwidth for the localisation and environmental monitoring services. For further details on this infrastructure see (Cavallo et al. 2014b). Two different data loggers collected the data on a Personal Computer, which acted as a gateway to send all data to the cloud platform. 
Fig. 4 The caregiver sets the event using a web portal; at a specific time, the robot reaches the user inside the house and acts as a physical reminder. The user then takes the medicine and sends feedback to the caregiver through the robot app

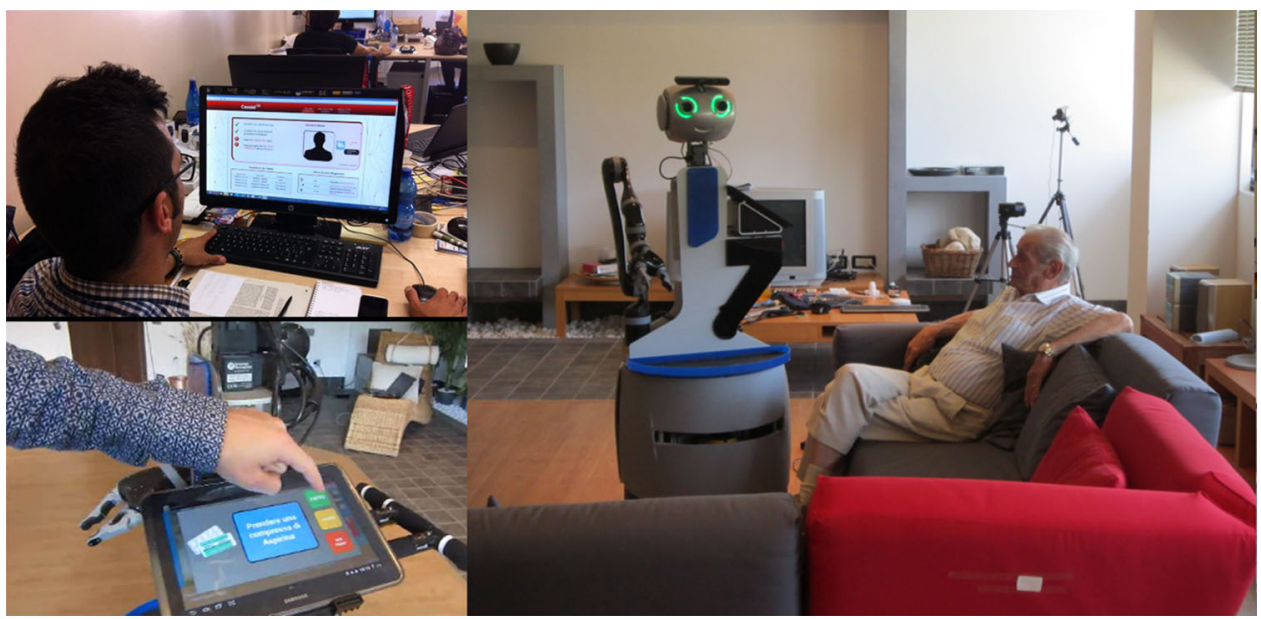

DoRo (Robot-Era; (Cavallo et al. 2014b) is a mobile robot designed on the SCITOS G5 platform (Metralabs, Germany) and equipped with a touch screen tablet, voice recognition and synthesis, and coloured Light Emitted Diodes in the eyes for facilitated and immediate interaction with the user (Fig. 4). It is also endowed with localisation and obstacle avoidance sensors for autonomous movement in the environment, a manipulator (Jaco, Kinova, Canada) and a Wi-Fi module to communicate with the cloud. The local control software of the robots was implemented by ROS nodes (Wang et al. 2013), while the execution of their functionalities was bounded to actions commanded by the reasoning module (Bangor et al. 2008).

Communication Layer Communication between hardware components and the hardware and the cloud infrastructure comprised two complementary tiers (Hu et al. 2012): Machine-to-Machine (M2M) and Machine-to-Cloud (M2C) communication modules. ZigBee, WI-FI and TCP/IP protocols were used for M2M communication to directly exchange data between the agents at the hardware layer. TCP/IP protocol connected the physical agents to the cloud in the M2C communications for data exchange and service delivery.

Application Layer The cloud platform provided SaaS features with the implementation of a DataBase (DB), a User Localisation Module (ULM) and an Event Scheduler Module (ESM).

The DB was conceptually divided into two different parts: one related to the sensors and one to the users. The list of the sensors installed in the wireless sensor network was reported in a specific entity. The unique sensor's EUI64 was used as a primary key of the n-upla that contains the sensor type (light, temperature, presence, etc.), the position in the environment (x,y coordinates), the calibration parameters if needed and the sensing workspace. For each i-th typology of the sensor, a specific entity collected sensor output over time. In the user part, all the information concerning the reminder part, such as the event scheduling for drug reminder and the user feedback, was reported. A DataBase Management Software (DBMS) managed DB entries and queries.

The ULM provided multi-user localisation service in large environments. The software used localisation data from the DB, implementing a sensor fusion approach based on a Kalman Filter for estimating the user's position. The KF was implemented exploiting both range-free (Arias et al. 2004) and range-based (Cavallo et al. 2014a) localisation methods, according to Wang et al. (Wang et al. 2013). Presence sensors were also used to improve positioning accuracy and perform host detection. A complete description and evaluation of ULM is reported in (Bonaccorsi et al. 2015).

The ESM, as a core engine deployed in the cloud, extrapolated events with sensorial reading, sent actions into the system depending on the perceived events and enabled notifications on the robot's tablet.

Interface Layer The interaction with the user was implemented by two different clients: 'Cassiel', a web portal for caregivers, and 'Ricordati 2.0', an Android App for older people (Fig. 5). In the design phase, security and privacy issues were respected using secure a connection via https with users being anonymous guaranteeing secure data transfer in the web.

Cassiel (Fig. 5a) was designed with different web pages which informal and formal caregivers could access through authentication and managing events, such as 'drug reminders', 'medical check-up' or 'ambient alerts'. The portal has two main sections: one to manage the feedback coming from the user and his/her environment, and another to schedule the pharmacologic therapy. Regarding medicine scheduling, the caregiver can organise the drug reminder events by setting different types of alerts. Practically, the portal presents a form that is filled in with information such as the type of event (e.g., unique or periodic), the time of alert (sometimes more than one during the day), the type of medicine and the dosage. In this way, the reminder service could be personalised according to the end user's needs and habits. Furthermore, Cassiel 
Fig. 5 The interface layer: a the Cassiel Web Portal, b Ricordati 2.0, the application installed on the DoRo tablet

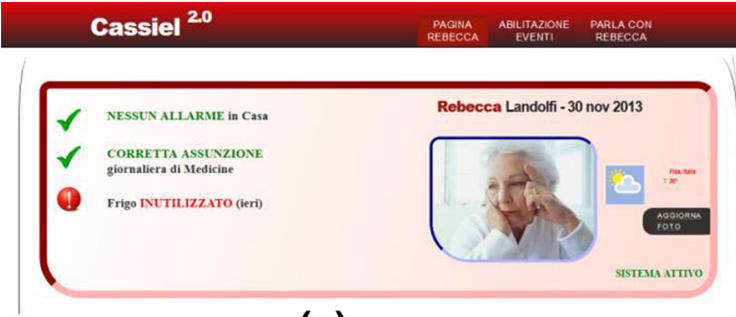

(a)

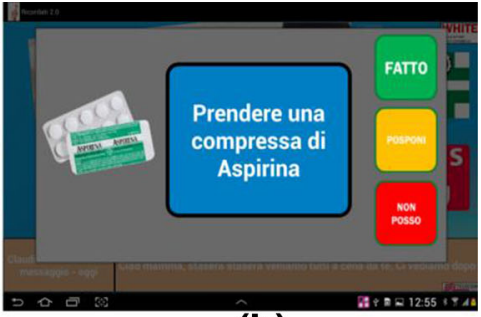

(b) accessed the DB, where all the data from the environmental network was stored, to extract several statistics about daily user-behaviour such as the number of times the person opens the entrance door at night and the average temperature, light and humidity.

Ricordati 2.0 (Fig. 5b) was an application installed on the robot's tablet and implemented with native android language. The application automatically starts when the tablet turns on and shows a slideshow of images (e.g., photos of beautiful moments of his/her life) inserted by the caregiver. At the bottom of the app home screen, the application presents the last message sent by the caregiver. This message appears in real time on the tablet using Firebase, a Platform as a Service (PaaS) in the cloud. The data sent to Firebase were JSON objects accessible via the RESTful service (Firebase). Moreover, when a reminder event occurs, Cassiel activates the reminder service. The robot reaches and alerts the user using a voice message. Furthermore, Ricordati 2.0 activates specific pop-ups. In order to enhance drug compliance, this pop-up shows an image of the medicine to take on the left and some keys on the right. These keys allow and oblige the users to leave feedback, such as 'Taken', 'Snooze' or 'I can't'. This feedback is reported on Cassiel web portal. As a consequence, the caregiver is informed about the correct/incorrect drug consumption for appropriate reactions.

\section{Results}

The data collected during the experimental phase were analysed, obtaining qualitative and quantitative results.

\subsection{Quality of service}

The RTT, conceived as the time needed by the web portal to receive the user's coordinates after the reminder event, was computed as the average time over the 20 experimental trials: the result was $56 \mathrm{~ms}$. In this implementation, the RPT was the time required by DoRo to plan the path to reach the user after receiving the user's coordinates and it was estimated to be $0.012 \mathrm{~ms}$. The TST was computed as the sum of RTT, RPT and ATT. TST strongly depended on the target positions of users, i.e. their distance from the robot and the robot's
Table 2 Average time over 20 trials to reach the target point.

\begin{tabular}{lclll}
\hline Case & Distance $(\mathrm{m})$ & ATT $(\mathrm{ms})$ & Mean velocity $(\mathrm{m} / \mathrm{s})$ & TST $(\mathrm{s})$ \\
\hline A & 3.6 & 16.35 & 0.22 & 16.38 \\
B & 8.2 & 21.90 & 0.37 & 21.93 \\
C & 12.6 & 40.06 & 0.31 & 40.08 \\
D & 10.9 & 25.41 & 0.42 & 25.43 \\
\hline
\end{tabular}

velocity. The complete results are shown in Table 2, where the mean velocity of the robot for each target is also shown.

\subsection{Acceptance of the service}

Data showed that the older volunteers were very familiar with home appliances and electronic devices for everyday use, such as TVs, DVD players and mobile phones. However, looking at the most current technological devices, such as PCs, smartphones or tablets, their knowledge fell short. As further proof, just 13 participants were able to correctly use a PC and the internet for entertainment and information.

The Cronbach's Alpha index was calculated to demonstrate the reliability of each construct. All constructs had an Alpha value higher than 0.70 , the cut-off value for being acceptable (Santos 1999), except Social Influence (SI) which was omitted from the analyses (see Table 3 ).

Regarding the usability and the acceptability, the participant's scores for each construct were added together and converted to a new score ranging from 0-100. The results showed that the proposed system was not usable for 4 older volunteers $(17.39 \%, M=38.13, S D=18.19)$, was usable for 7 of them $(30.44 \%, M=69.64, S D=2.67)$ and was excellent for 12 participants $(52.17 \%, M=98.33, S D=2.46)$. Furthermore 5 older volunteers $(21.73 \%, M=58.37, S D=$ 4.62) evaluated the system as not acceptable, it was acceptable for 16 of them $(69.57 \%, M=74.25, S D=5.92)$ and was excellent for 2 participants $(8.70 \%, M=94.84, S D=$ 4.23). Regarding user's satisfaction with the service, 1 participant $(4.35 \%)$ was D, $3(13.05 \%)$ were NAND, $7(30.43 \%)$ were $\mathrm{A}$ and $12(52.17 \%)$ were SA, thus the majority of the sample thought that they would like to use this hybrid robot-cloud system frequently. Further confirmation was that participants felt very confident using it; specifically, about 
Table 3 Constructs included in the questionnaire with Cronbach's Alpha and the descriptive statistics of the participants (\%).

\begin{tabular}{|c|c|c|c|c|c|c|c|}
\hline Code & Construct & $\begin{array}{l}\text { Cronbach's } \\
\text { alpha }\end{array}$ & $\begin{array}{l}\text { Strongly disagree } \\
\text { (SD) }(\%)\end{array}$ & $\begin{array}{l}\text { Disagree } \\
\text { (D) }(\%)\end{array}$ & $\begin{array}{l}\text { Neither agree nor } \\
\text { disagree (NAND) (\%) }\end{array}$ & Agree (A) (\%) & $\begin{array}{l}\text { Strongly agree } \\
\text { (SA) }(\%)\end{array}$ \\
\hline ANX & Anxiety & 0.728 & 52.17 & 39.13 & 8.70 & - & - \\
\hline $\mathrm{FC}$ & $\begin{array}{l}\text { Facilitating } \\
\text { conditions }\end{array}$ & 0.782 & 4.35 & 8.70 & 8.70 & 47.83 & 30.42 \\
\hline ITU & $\begin{array}{l}\text { Intention } \\
\text { to use }\end{array}$ & 0.927 & 13.04 & 4.35 & 8.70 & 39.13 & 34.78 \\
\hline PAD & $\begin{array}{l}\text { Perceived } \\
\text { adaptability }\end{array}$ & 0.983 & 8.70 & - & 4.35 & 4.35 & 82.60 \\
\hline PENJ & $\begin{array}{l}\text { Perceived } \\
\text { enjoyment }\end{array}$ & 0.780 & - & 8.70 & 17.39 & 39.13 & 34.78 \\
\hline USA & Usability & 0.836 & - & 8.70 & 17.39 & 43.48 & 30.43 \\
\hline LEAR & Learnability & 0.701 & 4.35 & 4.35 & 21.74 & 4.35 & 65.21 \\
\hline PS & $\begin{array}{l}\text { Perceived } \\
\text { sociability }\end{array}$ & 0.721 & 8.70 & 21.74 & 30.43 & 26.09 & 13.04 \\
\hline PU & $\begin{array}{l}\text { Perceived } \\
\text { usefulness }\end{array}$ & 0.821 & 13.04 & 13.04 & 26.09 & 21.74 & 26.09 \\
\hline SP & $\begin{array}{l}\text { Social } \\
\text { presence }\end{array}$ & 0.758 & 60.85 & 26.09 & 8.70 & 4.35 & - \\
\hline Trust & Trust & 0.839 & - & - & 4.35 & 8.70 & 86.95 \\
\hline
\end{tabular}

the ease of use, $3(13.05 \%)$ were SD, $1(4.35 \%)$ was D, 2 $(8.70 \%)$ were NAND, $4(17.40 \%)$ were A and $13(56.50 \%)$ were SA. Finally about the effectiveness, all participants were able to complete the task without help and in particular most of them $(65.22 \%)$ thought that they would be able to use this system without the support of a technical person and they did not think they would have to learn a lot of things before using it $(1(13.05 \%)$ was SD, $1(4.35 \%)$ was D, $2(8.70 \%)$ were NAND, 4 (17.40\%) were A and $13(56.50 \%)$ were SA).

Furthermore there was no significant correlation between age and usability score, and there was no effect of gender as shown in other studies (Abidi and Singh 2013).

Moreover the correlations analysis showed the strength of relations between constructs (See Fig. 6). In particular the proposed system was perceived as easy to learn (LEAR $65.21 \% \mathrm{SA}$ ) and to be adaptive to the changing needs of users (PAD $82.60 \% \mathrm{SA}$ ); thus these two aspects had an influence on the usability (LEAR-USA $r=0.780, p<.001$ ) (PAD-USA $r=0.462, p=0.002)$. Furthermore the USA construct correlated with the ANX one $(r=0.420, p=0.046)$, demonstrating that the high perceived usability did not fill the participants with anxiety. In effect older volunteers were very relaxed when using the hybrid robot-cloud system because 52.17 and $39.13 \%$ of them respectively strongly disagreed and disagreed with the questions concerning anxiety. Moreover a further confirmation of this was that the participants reported enjoyment associated with the use of the system (PENJ 39.13\% A and 34.78\% SA) and data showed an interrelation between USA and PENJ ( $r=0.416, p=0.049$ ).

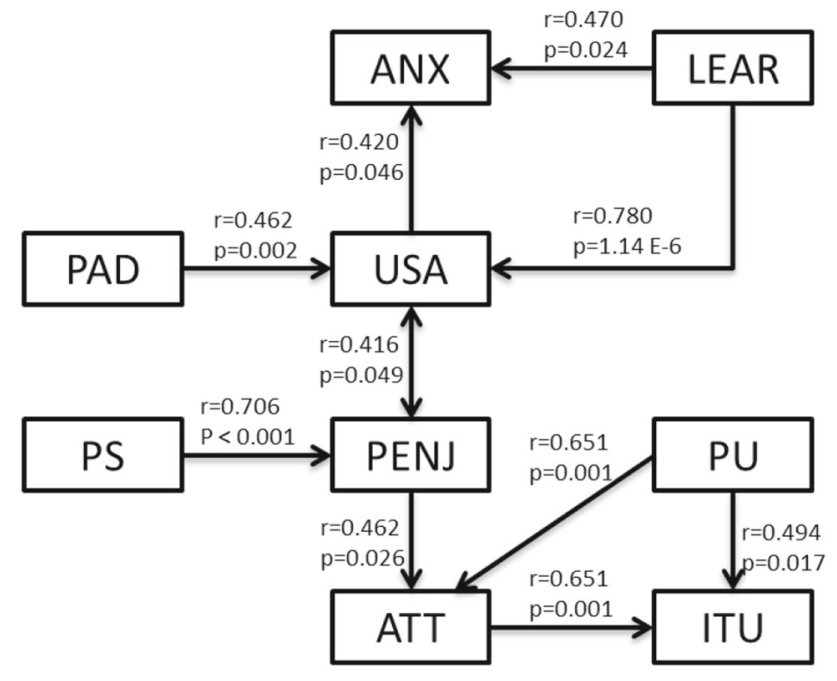

Fig. 6 Construct interrelations confirmed by correlation analysis; $r$ is the correlation value while $\mathrm{p}$ is the result of the statistical analysis

As expected the PS and PENJ constructs were strongly correlated $(r=0.706, p<.001)$; the perceived ability of the system to perform sociable behaviour was evaluated positively by many users (PS 26.09\% A and $13.04 \%$ SA). Furthermore the perceived enjoyment influenced the users' attitude $(r=0.462, p=0.026)$; the older people had a positive attitude towards the appliance of the hybrid robotcloud system in everyday life (ATT 34.78\% A and 26.09\% $\mathrm{SA}$ ). According to the survey, this robotic service was very 
useful for them (PU 21.74\% A and 26.09\% SA) influencing the feelings about the applicability of this technology $(r=0.651, p=0.001)$. Finally most of the participants were inclined to use the proposed system (ITU $39.13 \%$ A and $34.78 \% \mathrm{SA}$ ) especially in case of need and ITU constructs were directly driven by ATT $(r=0.651, p=0.001)$ and PU ( $r=0.494, p=0.017)$. In conclusion older users' positive feedback was confirmed by a high percentage (Trust $86.95 \%$ SA) believing that the system maintained personal integrity and reliability.

Concerning the comparison between different conditions and users, the Mann-Whitney $U$ and the Kruskal-Wallis tests were applied to analyse whether socio-demographic factors influenced acceptability. According to the results, older participants with a high educational level felt less anxiety than those with low education $(p=0.035)$ and those involved in other robotics experimentation such as DustCart (Ferri et al. 2011), Astromobile (Cavallo et al. 2014a) were more confident with the hybrid robot-cloud system ( $p=0.0524)$ because they were more familiar with the robotic systems.

\section{Discussion}

This study demonstrated the general feasibility of the integration of the system components over a cloud infrastructure to accomplish the reminder service. In this simplified implemented scenario, authors showed how the robot's computational abilities could be improved by external resources without increasing the computational cost of the robot. In particular, the unique capabilities 'on-board' exploited by DoRo reside in navigation and obstacle avoidance abilities. The cloud offloaded the database, drug reminders, and user localisation module, which provides an in-room user localisation sufficient for this service robotic application. For instance, even robots with limited abilities could be aware of user position; in this way, the robot can provide the service by going directly to the user.

The consequences of this achievement are not easily understood concerning a home care resident. On the contrary, the real societal impacts could be understood considering this hybrid robot-cloud model in institutionalised care arrangements. The caregivers can save working time demanded by the reminder service to the robot but maintain the supervision thanks to the web-portal and the user feedback. Consequently they can spend the saved time interacting/chatting with the patient improving the personal relationship thus avoiding feelings of loneliness and depression. From the robot's point of view, if it knows the position of the user, it can save time by avoiding looking for a specific user around the facilities and consequently can provide a higher number of reminders within a fixed time.
The robot's maximum velocity was pre-set at $0.6 \mathrm{~m} / \mathrm{s}$ to assure a safe approach and interaction with the user (Salvini et al. 2010). It was noticed that, during the service, the robot never reached this velocity because of the complexity of the real environment. In addition, the quantitative results highlighted how the proposed service has the potential to serve up to 30 requests each second.

The total service time depended mainly on the distance to the target. This guarantees an efficient service delivery compared with other QoS analyses with real users (Cavallo et al. 2014a). This quantitative results further emphasised the high system reliability and efficiency during the experimental phase, confirmed by the higher acceptability and usability results. Furthermore, the cloud offers more flexibility at a lower cost compared to dedicated servers (Abidi and Singh 2013).

In general, the proposed hybrid robot-cloud system was considered easy to use and well integrated by most of the older persons, demonstrating the usability of the system. Furthermore, considering the positive attitude towards the services and a high trust in the robot's ability, the acceptability was estimated to be good by older volunteers. Moreover there was a high ease of use, because most of the older participants were able to use the service without any external help.

Eventually, the proposed service could enhance the intensity and the duration of the pre-existing relationship between user/stakeholders and stakeholders/stakeholders. It reduces care discontinuity by exploiting a new kind of communication involving physical reminders.

In particular, from the user's point of view, this service enhances independent living, so the user can live alone in his/her own house as long as possible without decreasing treatment adherence. On the other hand, the user's family could be aware of the user's correct drug consumption and general health status. In this sense, the robot enhances the efficacy of the reminder service, thanks to its ability to move autonomously inside the house (Prakash et al. 2013). In addition, the family should be able to improve the coordination with other relevant stakeholders, such as pharmacists or elderly organisations, who could offer support in the care process.

As a consequence, companies have the opportunity to offer special healthcare management services by means of proprietary applications on the cloud. Besides, companies could be incentivised to develop specific cloud services by investigating new market opportunities and exploiting the proposed system. On the other hand, public administrations and municipalities should increase their efficacy and efficiency in the management of services for frail citizens in specific neighbourhoods.

Since privacy and security are not the main topics if this work, future research activities are already in schedule to involve these topics in cloud robotics field with a Crypto- 
botics approach, which aims to prepare these systems to be safely used in real applications (Morante et al. 2015).

\section{Conclusions}

This paper presented an innovative hybrid cloud solution for domiciliary reminder services in chronic disease management based on a mobile platform and a cloud platform delivering SaaS. The design and development were based on synergies coming from a multidisciplinary group of stakeholders (Moschetti et al. 2014), which allows for the analysis of the needs and requirements of all stakeholders and their relationships in order to design services and technological solutions that will effectively promote and support the idea of 'ageing well'.

This work mainly focused on the acceptability and usability from the primary user's point of view, i.e. older people; however the authors are well aware that personalised medical support for chronic disease management also includes other stakeholders, such as professional caregivers, family doctors, pharmacists, among others that will be investigated in future work.

Acknowledgements The authors thank the Cooperative 'Il Borgo', Peccioli, Italy and the Association 'Neurocare', Pisa, Italy for their valuable assistance with older persons' recruitment and for supervision during the experimental trials. Additionally, this work was supported by the European Community's 7th Framework Program (FP7-ICT-2011) under Grant agreement No. 288899 (Robot-Era Project) and grant agreement No.601116 (Echord++ project).

Open Access This article is distributed under the terms of the Creative Commons Attribution 4.0 International License (http://creativecomm ons.org/licenses/by/4.0/), which permits unrestricted use, distribution, and reproduction in any medium, provided you give appropriate credit to the original author(s) and the source, provide a link to the Creative Commons license, and indicate if changes were made.

\section{References}

Abidi, F., \& Singh, V. (2013, December). Cloud servers vs. dedicated servers-A survey. In 2013 IEEE international conference in MOOC innovation and technology in education (MITE) (pp. 1-5). IEEE.

AGE Platform Europe. (2010). European Charter European Charter of the rights and responsibilities of older people in need of long-term care and assistance. http://www.age-platform.eu/images/stories/ 22204_AGE_charte_europeenne_EN_v4.pdf.

Ajzen, I., \& Fishbein, M. (1980). Understanding attitudes and predicting social behaviour. Prentice-Hall.

Ajzen, I. (1991). The theory of planned behavior. Organizational Behavior and Human Decision Processes, 50(2), 179-211.

Arias, J., Zuloaga, A., Lázaro, J., Andreu, J., \& Astarloa, A. (2004). Malguki: Sn RSSI basedad hoc location algorithm. Microprocessors and Microsystems, 28(8), 403-409.

Auerbach, D. I. (2012). Will the NP workforce grow in the future? New forecasts and implications for healthcare delivery. Medical Care, 50(7), 606-610.
Badii, A., Etxeberria, I., Huijnen, C., Maseda, M., Dittenberger, S., Hochgatterer, A., et al. (2009). CompanionAble: Graceful integration of mobile robot companion with a smart home environment. Gerontechnology, 8(3), 181.

Bangor, A., Kortum, P. T., \& Miller, J. T. (2008). An empirical evaluation of the system usability scale. International Journal of HumanComputer Interaction, 24(6), 574-594.

Bonaccorsi, M., Fiorini, L., et al. (2015). Design of cloud robotic services for senior citizens to improve independent living in multiple. Environments. doi:10.3233/IA-150077.

Brooke, J. (1996). SUS-A quick and dirty usability scale. Usability evaluation in industry, 189(194), 4-7.

Cavallo, F., Aquilano, M., Bonaccorsi, M., Limosani, R., Manzi, A., Carrozza, M. C., \& Dario, P. (2014b). Improving domiciliary robotic services by integrating the ASTRO robot in an AmI infrastructure. In Gearing up and accelerating cross-fertilization between academic and industrial robotics research in Europe (pp. 267-282). Springer International Publishing.

Cavallo, F., Limosani, R., Manzi, A., Bonaccorsi, M., Esposito, R., Di Rocco, M., et al. (2014a). Development of a socially believable multi-robot solution from town to home. Cognitive Computation, 6(4), 954-967.

Coradeschi, S., Kristoffersson, A., Loutfi, A., Von Rump, S., Cesta, A., Cortellessa, G., \& Gonzalez, J. (2011). Towards a methodology for longitudinal evaluation of social robotic telepresence for elderly. In Human robot interaction.

Datta, C., Yang, H. Y., Tiwari, P., Kuo, I. H., \& MacDonald, B. A. (2011). End user programming to enable closed-loop medication management using a healthcare robot. Social Science.

Davis, F. D. (1989). Perceived usefulness, perceived ease of use, and user acceptance of information technology. MIS Quarterly, 319340.

Dillon. (2001). Ser acceptance of information technology. Encyclopedia of human factors and ergonomics. London: Taylor and Francis.

e-Pill Medication system, official website available at: http://www.epill. com.

EU Employment and Social Situation. (2013). Quarterly review, Special Supplement on demographic trend.

Eurostat. (2013). European social statistics-edition 2013. Available at: http://epp.eurostat.ec.europa.eu/cache/ITY_OFFPUB/KS-FP13-001/EN/KS-FP-13-001-EN.PDF.

Ferri, G., Manzi, A., Salvini, P., Mazzolai, B., Laschi, C., \& Dario, P. (2011, May). DustCart, an autonomous robot for door-to-door garbage collection: From DustBot project to the experimentation in the small town of Peccioli. In 2011 IEEE international conference on robotics and automation (ICRA) (pp. 655-660). IEEE. Firebase official website: https://www.firebase.com/.

Firebase official website: https://www.firebase.com/.

Goldberg, K. (2014). "Robots with their heads in the cloudsThe five elements of Cloud Robotics", Aspen Ideas Festival 2014. https://medium.com/aspen-ideas/robots-with-their-headsin-theclouds-e88ac44def8a.

Haynes, R. B., Ackloo, E., Sahota, N., McDonald, H. P., \& Yao, X. (2008). Interventions for enhancing medication adherence. Cochrane Database of Systematic Reviews, 2(2),

Healthdesk. (2014 Nov). "I pazienti e i farmaci, un rapporto da migliorare", Indagine civica sull'esperienza dei pazienti rispetto all'uso dei farmaci, con focus su biologici e biosimilari, http://www.healthdesk.it/sanit/i_pazienti_e_i_farmaci_un_ rapporto_da_migliorare/1415970600.

Heerink, M., Krose, B., Evers, V., \& Wielinga, B. (2009, September). Measuring acceptance of an assistive social robot: a suggested toolkit. In The 18th IEEE international symposium on robot and human interactive communication, 2009. RO-MAN 2009 (pp. 528 533). IEEE. 
Heerink, M., Kröse, B. J. A., Wielinga, B. J., \& Evers, V. (2006). Studying the acceptance of a robotic agent by elderly users. International Journal of Assistive Robotics and Mechatronics, 7(3), 33-43.

Hooker, R. S., Cawley, J. F., \& Everett, C. M. (2011). Predictive modeling the physician assistant supply: 2010-2025. Public Health Reports, 126(5), 708.

Hu, G., Tay, W. P., \& Wen, Y. (2012). Cloud robotics: architecture, challenges and applications. IEEE Network, 26(3), 21-28.

Iwata, H., \& Sugano, S. (2009, May). Design of human symbiotic robot TWENDY-ONE. In IEEE international conference on robotics and automation, 2009. ICRA'09 (pp. 580-586). IEEE.

Kaerlein, T. (2015). Minimizing the Human? Functional reductions of complexity in social robotics and their cybernetic heritage. In Social robots from a human perspective (pp. 77-88). Springer International Publishing.

Kamei, K., Nishio, S., Hagita, N., \& Sato, M. (2012). Cloud networked robotics. IEEE Network, 26(3), 28-34.

Kehoe, B., Patil, S., Abbeel, P., \& Goldberg, K. (2015). A survey of research on cloud robotics and automation. IEEE Transactions on Automation Science and Engineering, 12(2), 398-409.

Lieto, J. M., \& Schmidt, K. S. (2005). Reduced ability to self-administer medication is associated with assisted living placement in a continuing care retirement community. Journal of the American Medical Directors Association, 6(4), 246-249.

Marengoni, A., Angleman, S., Melis, R., Mangialasche, F., Karp, A., Garmen, A., et al. (2011). Aging with multimorbidity: A systematic review of the literature. Ageing Research Reviews, 10(4), 430-439.

McLellan, S., Muddimer, A., \& Peres, S. C. (2012). The effect of experience on System Usability Scale ratings. Journal of Usability Studies, 7(2), 56-67.

Meyer, J., Brell, M., Hein, A., \& Gessler, S. (2009). Personal assistive robots for AAL services at home-the florence point of view. Homepage: http://www.florence-project.eu.

Morante, S., Victores, J. G., \& Balaguer, C. (2015). Cryptobotics: Why robots need cyber safety. Frontiers in Robotics and AI, 2, 23.

Moschetti, A., Fiorini, L., Aquilano, M., Cavallo, F., \& Dario, P. (2014). Preliminary findings of the AALIANCE2 ambient assisted living roadmap. In Ambient assisted living (pp. 335-342). Springer International Publishing.

Mukai, T., Onishi, M., Odashima, T., Hirano, S., \& Zhiwei, L., (2008). Development of the tactile sensor system of a human-interactive robot RI-MAN. IEEE Transactions on Robotics, 24(2), 505-512.

Osterberg, L., \& Blaschke, T. (2005). Adherence to medication. New England Journal of Medicine, 353(5), 487-497.

Prakash, A., Beer, J. M., Deyle, T., Smarr, C. A., Chen, T. L., Mitzner, T. L., \& Rogers, W. A. (2013, March). Older adults' medication management in the home: How can robots help?. In 2013 8th ACM/IEEE international conference on human-robot interaction (HRI) (pp. 283-290). IEEE.

Robot, Operating System (ROS), official website available at: http:// www.ros.org/, visited on November 2014.

Robot-Era, Project, official website available at: http://www.robot-era. eu/robotera/index.php.

Rouse, M., Round Trip Time, Search Networking, available at: http:// searchnetworking.techtarget.com/definition/round-trip-time.

Salvini, P., Laschi, C., \& Dario, P. (2010). Design for acceptability: Improving robots' coexistence in human society. International Journal of Social Robotics, 2(4), 451-460.

Santos, J. R. A. (1999). Cronbach's alpha: A tool for assessing the reliability of scales. Journal of extension, 37(2), 1-5.

Sinčák, P., Loreník, D., Viríkova, M., \& Gamec, J. (2015). Emergent trends in robotics and intelligent systems (pp. 13-30). Springer International Publishing.
Smith, M., Bates, D. W., \& Bodenheimer, T. S. (2013). Pharmacists belong in accountable care organizations and integrated care teams. Health Affairs, 32(11), 1963-1970.

Socio Political Observatory in Europe. (2011). Elder care services, Available at: http://www.sociopolitical-observatory.eu.

Solis, P., \& Carlaw, S.(2013). Consumer and personal robotics. ABI Research. Philips Medication Dispensing Service, official website available at: http://www.managemypills.com/content/home.

Stiehl, W. D., Lieberman, J., Breazeal, C., Basel, L., Lalla, L., \& Wolf, M. (2005). The design of thehuggable: A therapeutic robotic companion for relational, affective touch. In $A A A I$ fall symposium oncaring machines: AI in Eldercare, Washington, DC.

Taipale, S., de Luca, F., Sarrica, M., \& Fortunati, L. (2015). Robot shift from industrial production tosocial reproduction. In Social robots from a human perspective (pp. 11-24). Springer InternationalPublishing.

Tiwari, P., Warren, J., Day, K. J., \& MacDonald, B. (2010). Some nontechnology implications for wider application of robots to assist older people. Health Care and Informatics Review Online.

Tiwari, P., Warren, J., Day, K., MacDonald, B., Jayawardena, C., Kuo, I. H., ... \& Datta, C. (2011, January). Feasibility study of a robotic medication assistant for the elderly. In Proceedings of the twelfth Australasian user interface conference- Vol. 117 (pp. 57-66). Australian Computer Society, Inc..

Venkatesh, V., Morris, M. G., Davis, G. B., \& Davis, F. D. (2003). User acceptance of information technology: Toward a unified view. MIS quarterly, 425-478.

Wahl, H. W., Iwarsson, S., \& Oswald, F. (2012). Aging well and the environment: Toward an integrative model and research agenda for the future. The Gerontologist. doi:10.1093/geront/gnr154

Wang, Y., Jin, Q., \& Ma, J. (2013, August). Integration of rangebased and range-free localization algorithms in wireless sensor networks for mobile clouds. In Green Computing and Communications (GreenCom), 2013 IEEE and internet of things (iThings/CPSCom), IEEE international conference on and IEEE cyber, physical and social computing (pp. 957-961). IEEE.

Zhang, W., Lu, H., Zhang, R., Xue, X., \& Weng, J. (2008, July). The architecture and body of FUWA developmental humanoid. In IEEE/ASME international conference on advanced intelligent mechatronics, 2008. AIM 2008. (pp. 1037-1040). IEEE.

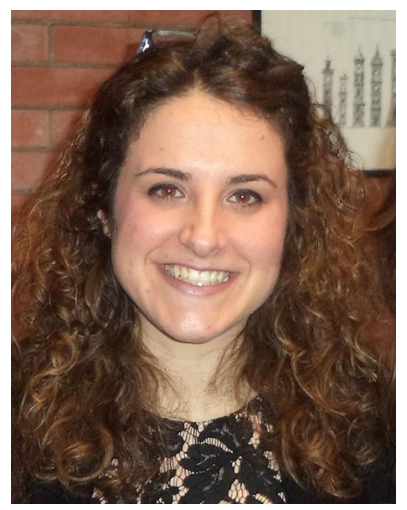

Laura Fiorini was awarded a M.Sc. in Biomedical Engineering (with honours) from the School of Engineering at the University of Pisa (IT) in 2012. To date, she is a Ph.D. student in Biorobotics at the BioRobotics Institute of Scuola Superiore Sant'Anna, Pisa. Her main research interests are in the fields of Ambient Assisted Living (AAL) and Service Robotics to prevent, support and enhance the quality of life of senior citizen. Actually, she is working on the design of a Cloud Robotics solutions for AAL applications. 

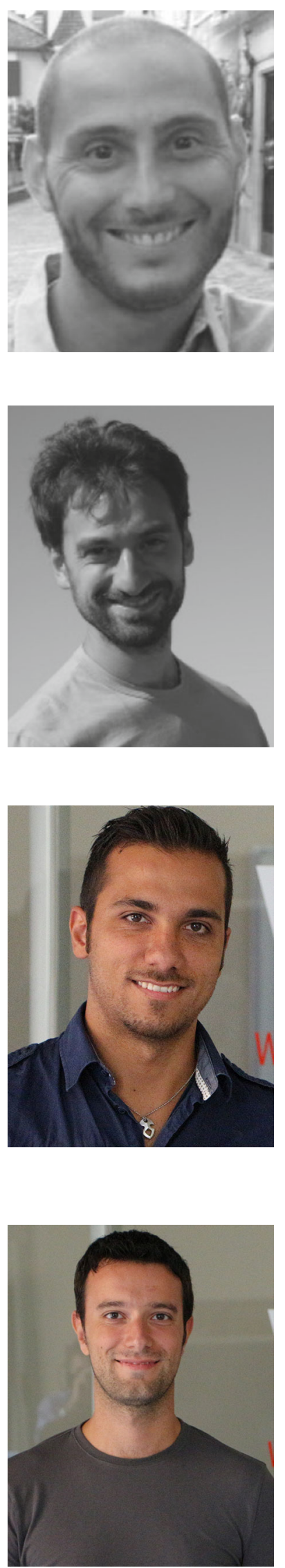

Raffaele Esposito was awarded a M.Sc. in Biomedical Engineering from the School of Engineering at the University of Pisa (IT) in 2009. To date, he is a research assistant at the BioRobotics Institute of Scuola Superiore Sant'Anna, Pisa. His main research interests are in the fields of usability, acceptability and dependability of Ambient Assisted Living (AAL) and Service Robotics solution to prevent, support and enhance the quality of life of senior citizens.

Manuele Bonaccorsi was graduated in Biomedical Engineering cum laude in 2010 at University of Pisa (IT)and defended his Ph.D. degree in BioRobotics (The BioRobotics Institute, SSSA) in 2014. Main research activities regarded the Ambient Assisted Living and indoor localization of people using wireless sensor networks to provide user position awareness to robotic platforms.

Claudio Petrazzuolo was graduated in Telecommunication Engineering from University of Naples "Federico II", earned a II level master's degree in ICT Innovation regarding innovative solutions and Cloud Computing. Currently, he is a researcher at WHITE lab in Pisa. He is aiming at developing innovative proofof-concepts, web-based applications and systems for elderly people and their caregivers in the Ambient Assisted Living (AAL) domain.

Filippo Saponara was graduated in Telecommunication Engineering from University of Naples "Federico II", earned a II level master's degree in ICT Innovation regarding innovative solutions and Cloud Computing. Actually he is a researcher at the WHITE lab in Pisa. His activities are focused on developing applications for tele-rehabilitation and Ambient Assisted Living with a focus on Android technology.

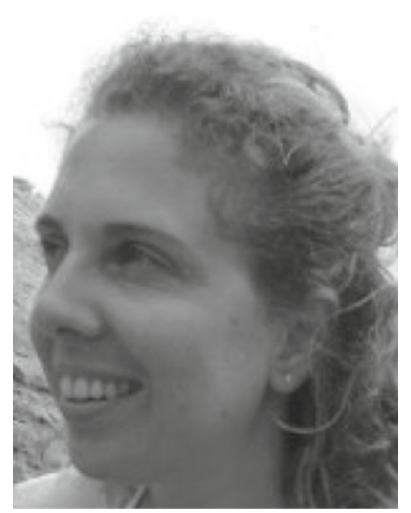

Roberta Giannantonio is graduated in Telecommunication Engineering at Politecnico di Torino in 2004 with a master thesis about multicast in ad hoc wireless networks that was carried out at Trinity College in Dublin, Ireland. Then, she joined Telecom Italia $\mathrm{Lab}$ and she has been involved in innovation projects about Wireless Technologies, mainly working on low power radio technologies (ZigBee, Bluetooth) and taking care of different application areas. In 2014 she started working into the SWARM Joint Open Lab of Telecom Italia on distributed and cooperative applications mainly based on mobile devices. She is author of patents and papers about wireless technologies.

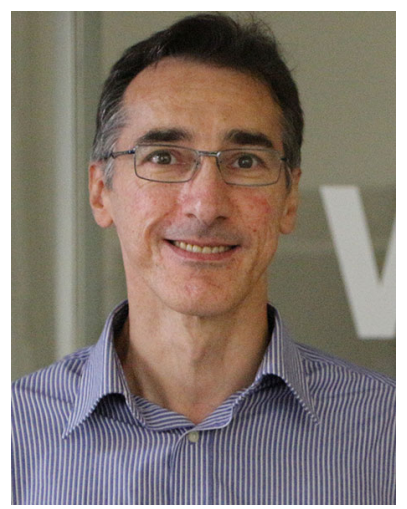

Gianluca De Petris is the director of the WHITE Lab in Pisa, graduated in Computer Science from University of Pisa in 1993, he earned a II level master's degree in Computer Science and Telecommunications from Politecnico di Torino in 1995 , and the PMP certification from PMI in 2010. Since 1st February, 1995 he is a researcher at Telecom Italia R\&D Center, in the area of "Technologies and Multimedia Services", since 2001 he is a Project Manager and coordinate several research projects. He is co-authors of patents and research papers.

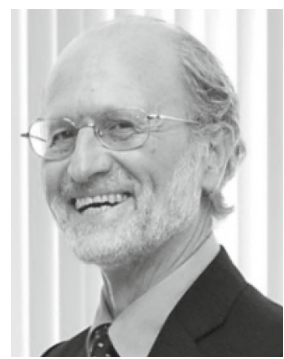

Paolo Dario is Professor of Biomedical Robotics and Director of The BioRobotics Institute of the Scuola Superiore Sant'Anna (SSSA), Pisa, Italy. He is the Coordinator of the Ph.D. Program in BioRobotics at SSSA, enrolling 85 Ph.D. students. He has been Founding Coordinator of the Center of MicroBioRobotics@SSSA of the Italian Institute of Technology (IIT), and he is currently Senior Scientist of IIT. He has been Visiting Professor at Brown University, Providence, RI, USA; at the École Polytechnique Fédérale de Lausanne (EPFL), Lausanne, Switzerland; at the École Normale Superieure de Cachan, France; at the College de France, Paris, France; at the Polytechnic University of Catalunya, Barcelona, Spain; at Waseda University, Tokyo, Japan; at Zhejiang University, Hangzhou and at Tianjin University, China.His main research interests are in the fields of medical robotics, bio-robotics, biomechatronics and micro/nano engineering and robotics. He is the coordinator of many national and European projects, the editor of two books on the subject of robotics, and the author of more than 500 scientific papers (300+ on ISI journals). 


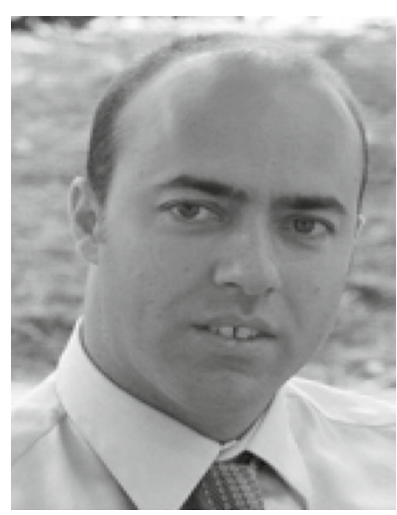

Filippo Cavallo MScEE, Ph.D.

in Bioengineering, is Assistant Professor at the BioRobotics Institute of Scuola Superiore Sant'Anna, (Pisa, Italy), focusing on cloud and social robotics, ambient assisted living, wireless and wearable sensor systems, biomedical processing, acceptability and ICT and AAL roadmapping. He participated in various National and European projects and currently is project manager of RobotEra, AALIANCE2 and Parkinson Project. He was visiting researcher at the the EndoCAS Center of Excellence, Pisa, Italy working in Computer Assisted Surgery (20052007); at the Takanishi Lab, Waseda University, Tokyo, Japan working on wireless sensor network (2007); at Tecnalia Recerch Center, Basque Country, Spain working on wearable sensor system for AAL. He is author of various papers on conferences and ISI journals. 